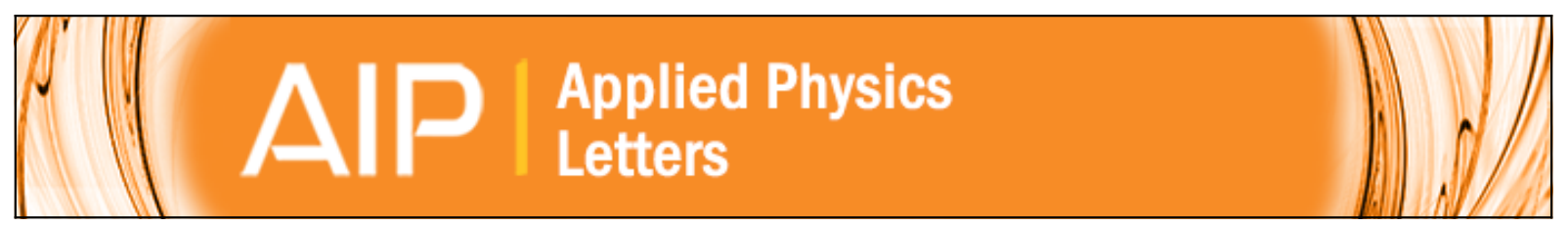

\title{
Softening of infrared-active mode of perovskite BaZrO3 proved by terahertz time- domain spectroscopy
}

M. A. Helal, T. Mori, and S. Kojima

Citation: Applied Physics Letters 106, 182904 (2015); doi: 10.1063/1.4919913

View online: http://dx.doi.org/10.1063/1.4919913

View Table of Contents: http://scitation.aip.org/content/aip/journal/apl/106/18?ver=pdfcov

Published by the AIP Publishing

\section{Articles you may be interested in}

Highly constrained ferroelectric [BaTiO3] $(1-\mathrm{x}) \Lambda /[\mathrm{BaZrO} 3] \mathrm{x} \wedge$ superlattices: X-ray diffraction and Raman spectroscopy

J. Appl. Phys. 116, 034108 (2014); 10.1063/1.4890513

Lattice dynamics and broad-band dielectric properties of the $\mathrm{KTaO} 3$ ceramics

J. Appl. Phys. 111, 104101 (2012); 10.1063/1.4714545

Ultrabroadband dielectric spectroscopy and phonons in ( Pb 1-x/ 2 La x ) ( Zr 0.9 Ti 0.1 ) O 3

J. Appl. Phys. 108, 104101 (2010); 10.1063/1.3501105

Ferroelectric BaTiO 3 / BaZrO 3 superlattices: X-ray diffraction, Raman spectroscopy, and polarization hysteresis loops

J. Appl. Phys. 108, 084104 (2010); 10.1063/1.3496620

Observation of TO 1 soft mode in $\mathrm{Sr} \mathrm{Ti} O 3$ films by terahertz time domain spectroscopy

Appl. Phys. Lett. 87, 182909 (2005); 10.1063/1.2128039

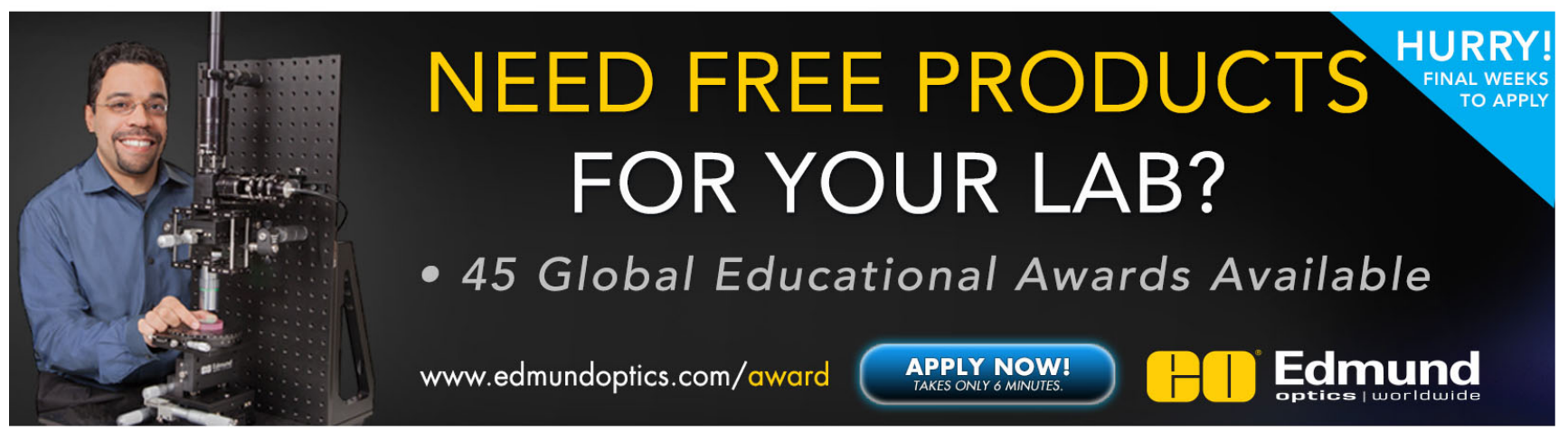




\title{
Softening of infrared-active mode of perovskite $\mathrm{BaZrO}_{3}$ proved by terahertz time-domain spectroscopy
}

\author{
M. A. Helal, ${ }^{\text {a),b) }}$ T. Mori, ${ }^{\text {b) }}$ and S. Kojima \\ Graduate School of Pure and Applied Sciences, University of Tsukuba, Tsukuba, Ibaraki 305-8573, Japan
}

(Received 3 March 2015; accepted 28 April 2015; published online 6 May 2015)

\begin{abstract}
The low-frequency infrared-active optical modes were studied in a barium zirconate, $\mathrm{BaZrO}_{3}$, single crystal with the perovskite structure using terahertz (THz) time-domain spectroscopy (TDS). The real and imaginary parts of the dielectric constants were accurately determined in the frequency range between 0.2 and $2.7 \mathrm{THz}$. Upon cooling from room temperature to $8 \mathrm{~K}$, the lowestfrequency TO1 mode at $2.32 \mathrm{THz}$ showed a pronounced softening to $1.94 \mathrm{THz}$. The real part of the dielectric constant at $0.5 \mathrm{THz}$ determined by THz-TDS obeys Barrett's relation, and the existence of a plateau confirms that the quantum effects lead to saturation of the soft mode frequencies of the TO1 and TO2 modes below $\approx 20 \mathrm{~K}$. This is reminiscent of incipient ferroelectrics with the perovskite structure such as $\mathrm{CaTiO}_{3}$. C 2015 AIP Publishing LLC. [http://dx.doi.org/10.1063/1.4919913]
\end{abstract}

The perovskite oxide family with the general formula $\mathrm{ABO}_{3}$ is one of the most important classes of multifunctional materials due to their superior physical properties and wide use in various technological applications, such as solid oxide fuel cells, steam electrolysis, multiferroics, and catalysts. ${ }^{1-3}$ There has been a great deal of interest and challenge to understand the behaviors of the dielectric properties of perovskite oxides, especially, investigating the relationship between the dielectric properties and the infrared-active phonon responses. ${ }^{4-6}$ However, the perovskite oxides with a cubic structure have a center of symmetry, and all the infrared-active modes are Raman inactive based on the selection rule. Although the low-frequency infrared-active modes play a dominant role in the dielectric properties, the experimental study in the far-infrared region has been insufficient.

In recent years, new interest has arisen in barium zirconate $\left(\mathrm{BaZrO}_{3}, \mathrm{BZO}\right)$ with the cubic perovskite structure due to its desirable properties as a high-temperature proton conductor, ${ }^{7}$ a dielectric material for use in wireless communications applications, ${ }^{8}$ and as a substrate for thin-film growth. ${ }^{9}$ Due to its large lattice constant, high melting point, low thermal expansion coefficient, low dielectric loss, and low thermal conductivity, BZO has become very attractive and popular not only for fundamental research but also for device applications. Recently, several theoretical and experimental investigations have been performed to explore the structural instability of cubic BZO. ${ }^{10-13}$ Unlike most perovskite systems, BZO does not undergo any structural phase transition and thus retains its cubic symmetry down to $2 \mathrm{~K}$. ${ }^{13}$

Recent developments in the technology of the generation and detection of terahertz $(\mathrm{THz})$ radiation have initiated a lot of attention for various applications such as $\mathrm{THz}$ imaging systems as well as the characterization of various materials in the $\mathrm{THz}$ frequency spectrum by $\mathrm{THz}$ time-domain spectroscopy (THz-TDS). ${ }^{14}$ Current THz-TDS measurements on a quantum paraelectric $\mathrm{KTaO}_{3}$ (KTO) revealed that the dielectric constant of KTO is highly correlated with the

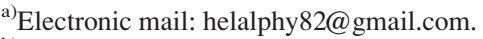

${ }^{b} \mathrm{M}$. A. Helal and T. Mori contributed equally to this work.
}

lowest infrared-active soft phonon mode at $2.09 \mathrm{THz}{ }^{15}$ Therefore, the study of the temperature dependence to explore whether the low-frequency infrared-active modes appear in BZO is important and also necessary.

In this study, we report the dielectric response of a BZO single crystal in the frequency range from $0.2 \mathrm{THz}$ to 2.7 THz using THz-TDS. Upon cooling, we found a clear softening of the lowest-frequency infrared-active transverse optical (TO) phonon mode near $2 \mathrm{THz}$.

The (001)-oriented BZO crystal plate with the dimensions of $3 \times 3 \mathrm{~mm}^{2}$ and thickness of $1 \mathrm{~mm}$ (Crystal Base Co. Ltd.) was used after polishing its surfaces to optical quality. The low frequency $\mathrm{THz}$ transmission spectra were measured using the THz-TDS system (Tochigi Nikon, RT-10000) in which the low temperature-grown GaAs photoconductive antennas were used for both the emitter and detector. These antennas were triggered by a mode-locked Ti:sapphire laser with a wavelength of $780 \mathrm{~nm}$, a pulse width less than $100 \mathrm{fs}$, and a repetition rate of $80 \mathrm{MHz} .^{16,17}$ In order to increase the $\mathrm{S} / \mathrm{N}$, the experimental data were the average of forty individual measurements.

Figure 1 shows the transmission power spectra of a reference and a BZO single crystal at $8 \mathrm{~K}$ at which a clear absorption peak at about $1.94 \mathrm{THz}$ is observed. According to the comparison of our THz-TDS data with those published in

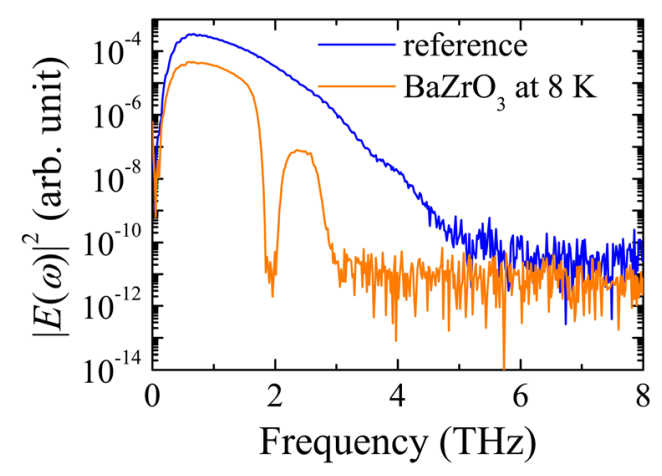

FIG. 1. The power spectra of a reference and a $\mathrm{BaZrO}_{3}$ crystal converted from time-domain waveforms at $8 \mathrm{~K}$. 
BZO ceramics by Nuzhnyy et al., ${ }^{18}$ we clearly observed another low-frequency mode near $2 \mathrm{THz}$. We also observed the same mode in the powder sample of BZO with the perovskite structure (Furuuchi Chemical Co. Ltd.). Nuzhnyy et al. ${ }^{18}$ used the nine oscillators model to fit the IR reflectivity spectra and they claimed that not all of the oscillators have a clear meaning. However, the appearance of the clear mode near $2 \mathrm{THz}$ in our measurements and the additional clear mode near $9 \mathrm{THz}$ in the $\mathrm{BZO}$ ceramics ${ }^{18}$ indicated that the $\mathrm{BZO}$ crystal has a lower symmetry than $P \mathrm{~m} \overline{3} \mathrm{~m}(\mathrm{Z}=1)$. The possible reason is that the ionic radius of a $\mathrm{Zr}$ ion (in BZO) is larger than that of the $\mathrm{Ti}$ ion (in $\mathrm{BaTiO}_{3}$ ), therefore, the instability may appear at the $R$-points, ${ }^{10}$ and the cubic lattice is slightly distorted thus causing the change from cubic $P \mathrm{~m} \overline{3} \mathrm{~m}$ to triclinic $P \overline{1} \quad(\mathrm{Z}=8)$. The $P \overline{1}$ symmetry of $\mathrm{BZO}$ has also been reported by Bennett et al. ${ }^{10}$ Since the number of atoms in the unit cell increases from 5 to 40 , the number of normal optical modes increases from 12 to 117 . Therefore, the observation of more lattice modes is reasonable in BZO. The lowest-frequency normal mode of the $P \overline{1}$ symmetry then appears at about $2 \mathrm{THz}$. The real $\left(\varepsilon^{\prime}\right)$ and imaginary $\left(\varepsilon^{\prime \prime}\right)$ parts of the dielectric constants in the THzTDS transmission spectra extracted from the time-domain waveforms at $8 \mathrm{~K}$ are plotted in Figs. 2(a) and 2(b) over the range from 0.2 to $2.7 \mathrm{THz}$.

In order to extract the mode frequency from the observed lowest TO1 mode, we fitted the spectra using the following damped harmonic oscillator (DHO) model:

$$
\varepsilon(\omega)=\varepsilon(\infty)+\sum_{j} \frac{\Delta \varepsilon_{j} \omega_{j}^{2}}{\omega_{j}^{2}-\omega^{2}-i \omega \Gamma_{\mathrm{j}}},
$$

where $\varepsilon(\infty)$ is the high frequency dielectric constant, and $\omega_{j}$, $\Delta \varepsilon_{j}$, and $\Gamma_{j}$ are the mode frequency, dielectric strength, and damping constant of each oscillator mode, respectively. We assumed two modes for the fitting. The parameters were

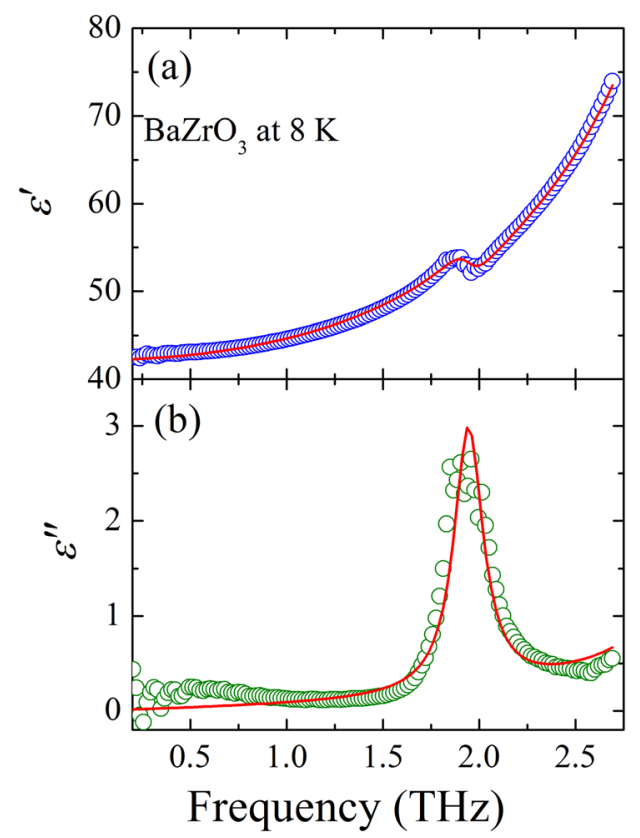

FIG. 2. (a) Real and (b) imaginary parts of complex dielectric constants of $\mathrm{BaZrO}_{3}$ at $8 \mathrm{~K}$. The solid lines denote the fitted lines with Eq. (1).
TABLE I. Values of fitting parameters of Eq. (1) for $\mathrm{BaZrO}_{3}$ at $8 \mathrm{~K}$.

\begin{tabular}{lcccccc}
\hline \hline$\varepsilon(\infty)$ & $\omega_{1}(\mathrm{THz})$ & $\omega_{2}(\mathrm{THz})$ & $\Delta \varepsilon_{1}$ & $\Delta \varepsilon_{2}$ & $\Gamma_{1}(\mathrm{THz})$ & $\Gamma_{2}(\mathrm{THz})$ \\
\hline 10 & 1.94 & 3.82 & 0.26 & 32.27 & 0.18 & 0.026 \\
\hline \hline
\end{tabular}

determined with a good accuracy by simultaneously fitting the real and imaginary parts of the dielectric constants, and the obtained parameters at $8 \mathrm{~K}$ are shown in Table I. Generally, the $\mathrm{THz}$ absorption is attributed to the interactions of the $\mathrm{THz}$ fields with the infrared-active lattice vibrations in a crystal. The optical phonon modes of the Brillouin zone center associated with the first-order dipole moment give rise to an intrinsic absorption due to lattice vibrations. The experimental data were in good agreement with the best fitted curves by Eq. (1) as shown by the solid lines in Figs. 2(a) and 2(b). This agreement indicated that the low-frequency $\mathrm{THz}$ response of $\mathrm{BZO}$ is mainly dominated by the TO1 and TO2 soft phonon modes. Generally, the IR-active phonons of BZO are the triply degenerate $T_{1 \mathrm{u}}$ modes, and the dielectric response is mainly ascribed to the contribution of the TO1 and the four phonon modes in Ref. 18. We speculate that the unstable acoustic phonon near the R-point of the Brillouin zone in a cubic lattice shows the phonon folding into the $\Gamma$-point of the distorted cubic structure which corresponds to the lowest-frequency TO1 mode. Therefore, the origin of the lowest-frequency TO1 mode near $2 \mathrm{THz}$ is not the Last mode at about $3.7 \mathrm{THz},{ }^{18}$ but the staggered oxygen octahedral tilts which have a significant effect on the Last mode. ${ }^{10}$ It couples to the soft TO2 mode with the same symmetry and shows softening together towards $T_{0}$. The temperature dependences of the TO1 mode frequency determined by fitting are shown in Fig. 3. A significant softening of the TO1 mode frequency is clearly observed upon cooling. Cochran predicted that the soft mode frequency varies with the temperature as

$$
\omega=\sqrt{A\left(T-T_{0}\right)},
$$

where $A$ is a constant and $T_{0}$ is the transition temperature. In Fig. 3, the soft mode frequency was well fitted by the green dashed line given by Eq. (2) above $20 \mathrm{~K}$. The parameters of this fit are $A=(7.82 \pm 0.2) \times 10^{-3} \mathrm{THz}^{2} \mathrm{~K}^{-1}$ and $T_{0}$ $=(-1020 \pm 22) \mathrm{K}$. However, the fitting line deviates from

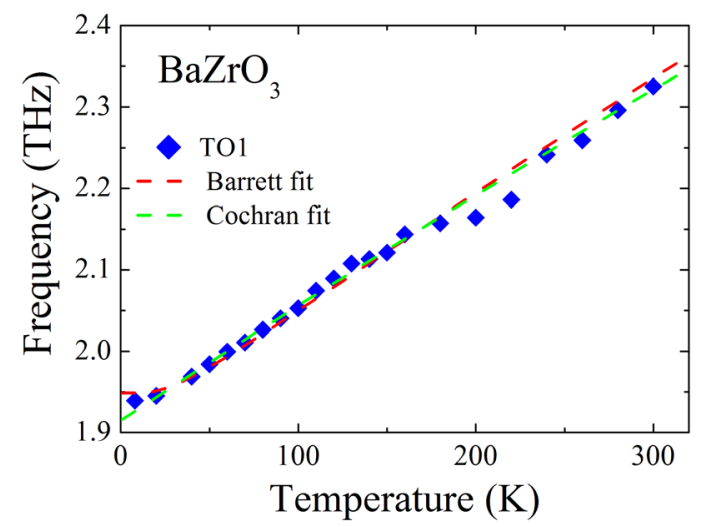

FIG. 3. Temperature dependences of TO1 mode frequency of $\mathrm{BaZrO}_{3}$. The green and red dashed lines denote the fitted lines with Eqs. (2) and (3), respectively. 
the experimental soft mode frequency below $20 \mathrm{~K}$. Therefore, assuming quantum fluctuations, it is better to apply the Barrett formula ${ }^{19}$

$$
\omega=\sqrt{A\left[T_{s} \operatorname{coth}\left(\frac{T_{s}}{T}\right)-T_{01}\right]},
$$

where $T_{\mathrm{s}}$ represents the saturation temperature. The fit with Eq. (3) in Fig. 3 by the red dashed line yields the following parameters: $A=(8.25 \pm 0.2) \times 10^{-3} \mathrm{THz}^{2} \mathrm{~K}^{-1}, T_{\mathrm{s}}=(40 \pm 5)$ $\mathrm{K}$, and so-called the classical Curie temperature, $T_{01}$ $=(-998 \pm 30) \mathrm{K}$. One can see that the transition temperatures in both cases have the same value within experimental error. This result confirms that BZO behaves as an incipient ferroelectric, which was previously observed in the incipient ferroelectric $\mathrm{CaTiO}_{3}$ (CTO). ${ }^{20}$ In quantum paraelectrics, the quantum zero-point motion prevents them from going into a ferroelectric phase. The quantum fluctuations suppress the softening of the low-frequency infrared-active modes, and the deviation from Eq. (2) occurs at very low temperatures. The high negative value of the extrapolated Curie temperature in both cases indicates that the stability of the paraelectric phase in $\mathrm{BZO}$ is higher than that of the quantum paraelectrics $\mathrm{SrTiO}_{3}$ $(\mathrm{STO})^{21}$ and KTO. ${ }^{22}$

The temperature dependence of $\varepsilon^{\prime}$ at $0.5 \mathrm{THz}$ is shown in Fig. 4(a). From 300 to $8 \mathrm{~K}, \varepsilon^{\prime}$ continuously increases with the decreasing temperature, attaining the value of $\varepsilon^{\prime} \approx 43$, a value that is slightly lower than that previously reported for a powder sample ${ }^{13}$ and it exhibits an overall saturation to a plateau below $\approx 20 \mathrm{~K}$. It is a characteristic feature of an incipient ferroelectric that the dielectric constant remains high and roughly constant for $T \rightarrow 0 \mathrm{~K}$ (Ref. 21) due to the fact that quantum fluctuations stabilize the paraelectric phase.

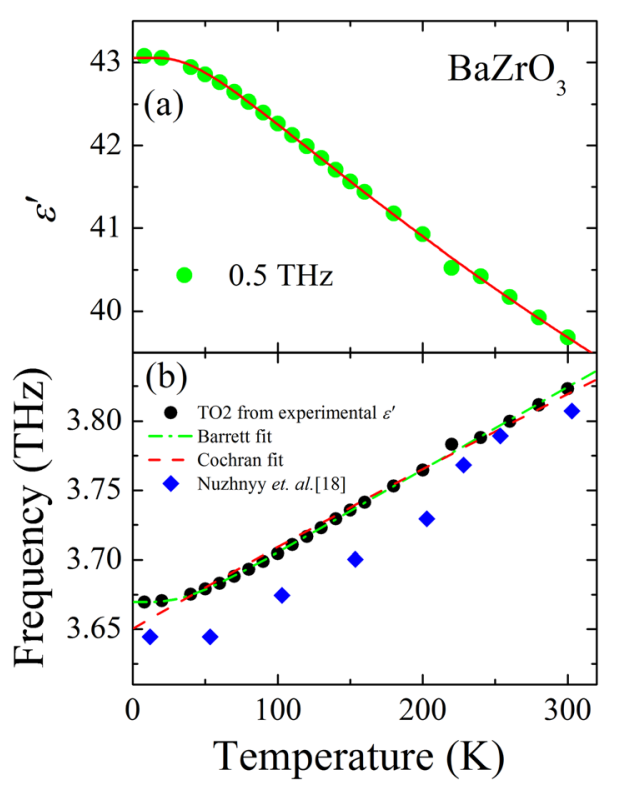

FIG. 4. (a) Temperature dependence of the real part $\varepsilon^{\prime}$ of complex dielectric constants at $0.5 \mathrm{THz}$ for $\mathrm{BaZrO}_{3}$. The red solid line represents the fitted line by Eq. (4). (b) Temperature dependence of TO2 mode frequency (filled circles) obtained from $\varepsilon^{\prime}$ at $0.5 \mathrm{THz}$ and LST relation (see text for details). The red dashed and green short dashed lines represent the fitted lines with Eqs. (2) and (3), respectively. The blue diamond represents the soft mode frequency extracted from Ref. 18 .
The existence of such a plateau has already been reported for the $\mathrm{STO}^{21}$ and $\mathrm{KTO}^{22}$ quantum ferroelectrics, and usually thought to be associated with the zero point vibration. In order to describe the temperature dependence of the dielectric constant of quantum paraelectrics, Barrett extended Slater's mean-field theory by including the quantum effect. ${ }^{23}$ Therefore, for a quantitative analysis, we fitted the temperature dependence of the real part of the dielectric constant in Fig. 4(a) using Barrett's relation ${ }^{23}$

$$
\varepsilon^{\prime}=B+\frac{C}{T_{s} \operatorname{coth}\left(\frac{T_{s}}{T}\right)-T_{01}},
$$

where $C$ and $T_{\mathrm{s}}$ can be recognized as the Curie constant and saturation temperature, respectively. ${ }^{21,22} B$ is a constant and temperature independent. It can be seen from Fig. 4(a) that the fitting data are in good agreement with the experimental data, and we obtained $C=(2.24 \pm 1.07) \times 10^{4} \mathrm{~K}, \quad T_{01}=(-1090 \pm 310) \mathrm{K}$, $T_{\mathrm{s}}=(64.0 \pm 5.9) \mathrm{K}$, and $B=(23.6 \pm 4.2)$. To determine the TO2 mode frequency from the experimental $\varepsilon^{\prime}$ at $0.5 \mathrm{THz}$, plotted in Fig. 4(a), we use the Laddane-Sachs-Teller (LST) relationship

$$
\varepsilon(0)=\varepsilon(\infty) \prod_{j}^{N} \frac{\omega_{L O j}^{2}}{\omega_{T O j}^{2}}
$$

where $\varepsilon(0)$ is the static dielectric constant, and $\omega_{L O j}$ and $\omega_{T O j}$ are the angular frequencies of the longitudinal and transverse optical phonon modes, respectively. Here, we assumed that $\varepsilon(0)$ is equivalent to $\varepsilon^{\prime}$ at $0.5 \mathrm{THz}$. In Fig. 4(b), the TO2 mode frequency (filled circles), calculated according to Eq. (5) with the experimental $\varepsilon^{\prime}$ at $0.5 \mathrm{THz}$ and the theoretical phonon frequencies obtained from Ref. 24, is plotted as a function of the temperature in comparison to the soft mode frequency obtained from BZO ceramics ${ }^{18}$ (blue diamonds). It is seen that our calculated TO2 mode frequency shows a significant softening upon cooling similar to the previously observed BZO ceramics. ${ }^{18}$ The TO2 soft mode vibration will be the Last type, i.e., vibration of $\mathrm{Ba}$ vs. $\mathrm{ZrO}_{6}$ as reported by Nuzhnyy et al. ${ }^{18}$ In order to have a better analysis, we fitted this TO2 mode frequency using Eq. (2) (green dashed line in Fig. 4(b)) and obtained the following parameters: $A$ $=(1.45 \pm 0.3) \times 10^{-3} \mathrm{THz}^{2} \mathrm{~K}^{-1}$ and $T_{0}=(-1001 \pm 26) \mathrm{K}$. Since the quality of fit is poor below $20 \mathrm{~K}$, therefore, we used Eq. (3) (red dashed line in Fig. 4(b)) which yields the fitting parameters of $A=(1.96 \pm 0.2) \times 10^{-3} \mathrm{THz}^{2} \mathrm{~K}^{-1}, T_{01}$ $=(-1090 \pm 17) \mathrm{K}$, and $T_{\mathrm{s}}=(54 \pm 4) \mathrm{K}$. The values of $T_{0}$ and $T_{01}$ are strongly negative in both the fitting data of the temperature dependence of the frequency softening and real part of the dielectric constant in comparison to the typical quantum paraelectrics, STO and KTO. In CTO, the dielectric constant increases with the decreasing temperature and saturates at low temperatures. According to this behavior, CTO has been classified as an incipient ferroelectric. ${ }^{20}$ Therefore, BZO is an incipient ferroelectric with a perovskite structure similar to CTO. $^{20}$

In conclusion, we have investigated the temperature dependence of the far-infrared dielectric properties of a BZO single crystal by THz-TDS. By comparing with previously 
reported data in BZO ceramics ${ }^{18}$ and first principles calculations, ${ }^{10}$ we propose that the lowest-frequency TO1 mode near $2 \mathrm{THz}$ is due to the $P \overline{1}$ symmetry of the BZO single crystal. The origin of the lowest TO1 mode, which has a low dielectric strength, can be attributed to the staggered arrangement of the oxygen octahedral, which couples to the soft TO2 mode and shows softening together towards $T_{0}$. The low-frequency TO1 and TO2 infrared-active phonon modes exhibit a significant softening and the dielectric constant increases upon cooling. Their temperature dependence accounts for the characteristics of other incipient ferroelectrics, such as CTO, in agreement with earlier dielectric studies. The real part of the complex dielectric constant at 0.5 THz obeys Barrett's relation, and the existence of the plateau confirms that the quantum effects lead to saturation of the soft mode frequencies of the TO1 and TO2 modes below $\approx 20 \mathrm{~K}$.

This work was partially supported by Grants-in-Aid for Young Scientists (B) (No. 24740194) from JSPS and the Casio Science Promotion Foundation.

${ }^{1}$ G. Tsekouras and J. T. S. Irvine, J. Mater. Chem. 21, 9367 (2011).

${ }^{2}$ J. Varghese, W. Whatmore, and J. D. Holmes, J. Mater. Chem. C 1, 2618 (2013).

${ }^{3}$ K. Maeda and K. Domen, Angew. Chem., Int. Ed. 51, 9865 (2012).

${ }^{4}$ A. S. Barker, Jr. and M. Tinkham, Phys. Rev. 125, 1527 (1962).

${ }^{5}$ W. G. Spitzer, R. C. Miller, D. A. Kleinman, and L. E. Howarth, Phys. Rev. 126, 1710 (1962).
${ }^{6}$ T. Ostapchuk, J. Petzelt, V. Zelezny, A. Paskin, J. Pokorny, I. Drbohlav, R. Kuzel, D. Rafaja, B. P. Gorshunov, M. Dressel, Ch. Ohly, S. Hoffmann-Eifert, and R. Waser, Phys. Rev. B 66, 235406 (2002).

${ }^{7}$ K. D. Kreuer, Annu. Rev. Mater. Res. 33, 333 (2003).

${ }^{8}$ M. T. Sebastian, Dielectric Materials for Wireless Communication (Elsevier, Amsterdam).

${ }^{9}$ P. S. Dobal, A. Dixit, R. S. Katiyar, Z. Yu, R. Guo, and A. S. Bhalla, J. Appl. Phys. 89, 8085 (2001).

${ }^{10}$ J. W. Bennett, I. Grinberg, and A. M. Rappe, Phys. Rev. B 73, 180102 (2006).

${ }^{11}$ A. Bilic and J. D. Gale, Phys. Rev. B 79, 174107 (2009).

${ }^{12}$ C. Zhu, K. Xia, G. R. Qian, C. L. Lu, W. Z. Luo, K. F. Wang, and J.-M. Liu, J. Appl. Phys. 105, 044110 (2009).

${ }^{13}$ A. R. Akbarzadeh, I. Kornev, C. Malibert, L. Bellaiche, and J. M. Kiat, Phys. Rev. B 72, 205104 (2005).

${ }^{14} \mathrm{G}$. Gruner, Millimeter and Submillimeter Wave Spectroscopy of Solids (Springer-Verlag, Berlin Heidelberg, 1998).

${ }^{15}$ Y. Ichikawa, M. Nagai, and K. Tanaka, Phys. Rev. B 71, 092106 (2005).

${ }^{16}$ T. Mori, H. Igawa, and S. Kojima, IOP Conf. Ser.: Mater. Sci. Eng. 54, 012006 (2014).

${ }^{17}$ H. Igawa, T. Mori, and S. Kojima, Jpn. J. Appl. Phys., Part 1 53, 05 FE01 (2014).

${ }^{18}$ D. Nuzhnyy, J. Petzelt, M. Savinov, T. Ostapchuk, V. Bovtun, M. Kempa, J. Hlinka, V. Buscaglia, M. T. Buscaglia, and P. Nanni, Phys. Rev. B 86, 014106 (2012).

${ }^{19}$ V. Goian, S. Kamba, J. Hlinka, P. Vanêk, A. A. Belik, T. Kolodiazhnyi, and J. Petzelt, Eur. Phys. J. B 71, 429 (2009).

${ }^{20}$ V. Zelezny, E. Cockayne, J. Petzelt, M. F. Limonov, D. E. Usvyat, V. V. Lemanov, and A. A. Volkov, Phys. Rev. B 66, 224303 (2002).

${ }^{21}$ K. A. Muller and H. Burkard, Phys. Rev. B 19, 3593 (1979).

${ }^{22}$ C. Ang, A. S. Bhalla, and L. E. Cross, Phys. Rev. B 64, 184104 (2001).

${ }^{23}$ J. H. Barrett, Phys. Rev. 86, 118 (1952).

${ }^{24}$ R. A. Evarestov, Phys. Rev. B 83, 014105 (2011). 